Postprint: Das P, Van Gelder L, Janssen H, Roels S. 2017. Designing uncertain optimization schemes for the economic assessment of stock energy-efficiency measures, Journal of Building

\title{
Designing uncertain optimization schemes for the economic assessment of stock energy-efficiency measures
}

\author{
Payel Das ${ }^{\mathrm{a}, \mathrm{b} *}$, Liesje Van Gelder ${ }^{\mathrm{c}}$, Hans Janssen ${ }^{\mathrm{c}}$, Staf Roels ${ }^{\mathrm{c}}$ \\ ${ }^{a}$ Rudolf Peierls Centre for Theoretical Physics, 1 Keble Road, Oxford, OX1 3NP, UK; \\ ${ }^{b} U C L$ Institute for Environmental Design and Engineering, Central House, 14 Upper Woburn Place, \\ London, WC1H ONN, UK; \\ ${ }^{c}$ Building Physics Section, Department of Civil Engineering, KU Leuven, Kasteelpark Arenberg 40 Bus \\ 2447, 3001 Heverlee, Belgium
}

()

\begin{abstract}
There are a myriad of options when it comes to designing an uncertain optimization scheme. This work explores the impacts of several of these options using a case-study retrofitting of Swedish attics developed during the Annex 55 project of the International Energy Agency Energy Buildings and Community programme.

We find that not considering maintenance costs, increments in energy costs with time, and discount rates in the economic criterion can impact the derived optimal design option. However for our case study no difference was found between criteria that examined absolute returns, fractional returns, and annulized returns, despite emphasising different aspects of the investment. If multiple moments of the economic criterion distribution are combined with weights to create a single objective function then several weights should be explored. Alternatively a single moment could be sought, such as the probability that the economic criterion is negative as a sufficient single objective, avoiding having to choose a weight. A hybrid genetic algorithm may offer a more efficient method of determining the optimal design option but a multi-layered sampling scheme offers more flexibility regarding subsequent analysis.
\end{abstract}

Keywords: energy efficiency, stock, building simulation, optimization, uncertainty

\section{Introduction}

Uncertain optimization entails the determination of an optimal set of parameters through the construction of an objective function that takes into account variabilities and uncertainties in the chosen performance criterion. In building performance simulations, it can be used to derive the optimal retrofitting scenario that reduces energy use without an adverse impact on durability or comfort, whilst also considering variabilities in dwelling characteristics across a stock, weather parameters, future economic scenario, and workmanship. Designing an uncertain optimization scheme for retrofitting dwellings requires the specification of several steps, once the parameters of the design options have been established. A choice needs to be made regarding the performance criterion for comparing the various design options, the derivation of single or multiple objective functions from a probabilistic performance criterion, and a scheme for sampling variabilities and generating design options.

Choosing an economically-driven performance criterion is a natural choice for energy-efficiency interventions, enabling the decision maker to easily compare different options. Choices for such a criterion include simply the cost of energy savings, or the payback period, which is usually given by the number of years for the initial investment to be repaid through energy savings. The Net Present Value $(N P V)$ gives a current 'value' for the intervention after a chosen number of years according to the initial investment, maintenance costs, and energy savings, all subject to a future

*Corresponding author. Email: payel.das@physics.ox.ac.uk 
Postprint: Das P, Van Gelder L, Janssen H, Roels S. 2017. Designing uncertain optimization schemes for the economic assessment of stock energy-efficiency measures, Journal of Building Performance Simulation, 10:3-16. doi: 10.1080/19401493.2015.1099054

change in energy price and discount rate (e.g. Malatji et al. 2013; Turner et al. 2013). Where the number of years is taken to be the lifespan of the intervention, it becomes a life cycle analysis (e.g. Tuhus-Dubrow and Krarti 2010; Fesanghary et al. 2012). There are several variants of this criterion: it may be normalised by the initial investments costs and maintenance costs (Return on Investment (e.g. Kuckshinrichs et al. 2010)) or instead the discount rate could be sought such that the the $N P V$ of an intervention after the chosen number of years is zero (Internal Rate of Return, e.g. Goodacre et al. (2002)).

In a deterministic optimization, the objective function would be simply equal to the deterministic performance criterion. However, if each design option is associated with a performance criterion distribution, a deterministic objective function(s) has to be derived to carry out a single (multi)-objective optimization. This is done by looking at moments of the performance criterion distribution. For example, the mean of the distribution is a measure of how well the design parameters do overall, and the dispersion is a measure of the variability in the results over a stock. Once the moments have been selected, in the case of a single moment, a single-objective optimization can be carried out. If several moments are considered, then they can either be suitably weighted and summed to create a single objective function (Das et al. 2013; Asadi et al. 2012) or particularly when they are conflicting, they can be left as multiple objectives to carry out a multi-objective optimization. This enables the resolving of the Pareto-optimal front (Das et al. 2014a), which enables a set of design options to be derived for which one objective cannot be improved without the other worsening (Deb 2001).

Finally, there are several different types of algorithms for exploring the multi-variate design space, including gradient-based methods such as the Levenberg-Marquardt algorithm, derivative-free direct search methods such as the systematic evaluation of evenly-spaced points covering the design space, and derivative-free stochastic algorithms that range from random sampling to evolutionary algorithms and Bayesian methods. Uncertain performance criteria have been combined with optimization algorithms in a handful of studies (Nguyen et al. 2014). Examples include the integration of the Latin Hypercube sampling method into a multi-layered sampling scheme. This samples design options in the first layer and then samples variabilities and uncertainties associated with the performance criterion distribution in subsequent layers (Van Gelder et al. 2014b). Rysanek and Choudhary (2013) present classical decision theories as methods of non-probabilistic optimisation under both technical and economic uncertainty. Booth et al. (2013) combines a Bayesian method for estimating uncertainty, Monte Carlo simulations for propagating uncertainty and then various economic criteria for exploring the design options, without attempting a formal optimization. Hopfe et al. (2013) explores how best to define weights between several objectives in the case of uncertain optimization by engaging directly with stakeholders.

It is clear from the literature that there are several choices to make when designing an uncertain optimization scheme. The aim of this paper is to explore the impacts of several of these choices to help the reader create an uncertain optimization scheme, suited to their particular design problem. In the process, we also hope to highlight the obvious advantages of using an uncertain optimization scheme rather than a deterministic scheme. The work is carried out in the context of a probabilistic economic analysis for a case study introduced in the framework of the Annex 55 project of the International Energy Agency (IEA) Energy Buildings and Community (EBC) programme ${ }^{1}$. The case study is introduced in Section 2, the variants in the uncertain optimization methodology are described in Section 3, the impact of different formulations are presented in Section 4, and discussed in Section 5. Finally, the results and conclusions of this work are summarised in Section 6.

\footnotetext{
${ }^{1}$ http://www.ecbcs.org/annexes/annex55.htm
} 
Postprint: Das P, Van Gelder L, Janssen H, Roels S. 2017. Designing uncertain optimization schemes for the economic assessment of stock energy-efficiency measures, Journal of Building Performance Simulation, 10:3-16. doi: 10.1080/19401493.2015.1099054

\section{Annex 55 case study}

The work presented in this paper is based on a case study developed for the IEA EBC Annex 55 project. The background to the project, the building simulation tool, potential energy efficiency measures for the case-study housing stock, and the construction of a surrogate model are shortly described here. More details can be found in the Subtask 2 report.

\subsection{Background}

The aim of Annex 55 was to develop probability-based design strategies in the retrofitting of buildings to reliably gauge the likelihood of success. The methods presented here were primarily assembled in Subtask 2, whose particular objective was to develop probabilistic tools for the calculation of energy use, life cycle cost, and hygrothermal performance. This covered the exploration of methods of uncertainty propagation (Janssen 2013), sensitivity analysis (Das et al. 2014b), metamodelling construction (Van Gelder et al. 2014a), and economic optimization (Van Gelder et al. 2014b). The final exercise of Subtask 2 aimed at synthesising the various methods to develop a framework for economic optimization that considered the propagation of uncertainty.

The methods were illustrated on cold attics in Sweden as a case study, which have suffered from high humidity levels over the last decade, resulting in significant mould growth on the wooden underlay of attic eaves (Ahrnens and Borglund 2007). Houses have been increasingly retrofitted with additional attic insulation, which leads to a colder attic space and hence a higher humidity (Hagentoft et al. 2008). The problem is further exacerbated by the ingress of moist indoor air from the indoor living area, cooling of the underside of the roof due to sky radiation, and water vapour in the encompassing outdoor air. Therefore it is paramount to provide the ideal rate of air exchange between the attic and outdoor air. If the air exchange rate is too high, and there is significant cooling of the wooden underlay, relativity humidity levels may be high as a result of moisture in the outdoor air. If the air exchange rate is too low and the attic floor is leaky, then humidity levels may be high as a result of moisture in the indoor air.

\subsection{The cold attic model}

The tool used to model hygrothermal conditions in the attics is a piece of MATLAB code initially created by Carl-Eric Hagentoft (Hagentoft 2011), and subsequently further developed in the duration of Annex 55. The attic (Figure 1) is assumed to have a roof with two identical low-sloped eaves, constructed from water-tight wooden underlay boards. The roof eaves are assumed to have an effective leakage area located in the junction between the roof and façade, and the heat capacity of the entire roof is assumed to be in the wooden underlay. The attic floor connects the attic to the indoor living area and has both thermal insulation and some level of airtightness. The heat balance equations assume a resultant air temperature in the attic $\left(T_{\mathrm{a}},{ }^{\circ} \mathrm{C}\right)$ that is dependent on heat exchange with outdoor air via leakage to the outside (conductance $K_{\mathrm{wl}}$, W/K), and indoor air through air leakage $\left(K_{\text {floorl }}\right)$ and heat transmission $\left(K_{\text {floor }}\right)$ through the attic floor. The temperature at the wooden underlay centre $\left(T_{\mathrm{u}}\right)$ is determined by heat exchange with the roof, which has temperature $T_{\text {eq }}$ and conductance $K_{\text {roof,se }}$. The thermal network of the attic is shown in Figure 1a. The moisture balance equations assume that the moisture storage capacity of the roof is also all located in the wooden underlay. The outer surface of the wooden underlay in the roof is assumed to be perfectly moisture tight and diffusion of moisture through the attic floor is neglected. The moisture content in the attic $\left(v_{\mathrm{a}}, \mathrm{kg} / \mathrm{m}^{3}\right)$ depends only on exchange with outdoor air through leakage in the attic walls (vapour conductance $k_{\mathrm{w}}, \mathrm{m}^{3} / \mathrm{a}$ ) and exchange with indoor air through leakage in the attic floor $\left(k_{\text {floor }}\right)$. The moisture content in the wooden underlay $\left(v_{\mathrm{u}}\right)$ is then determined by the moisture content in the attic and the vapour resistance $\left(k_{\mathrm{si}}\right)$ between the air and centre of the wooden underlay. The moisture network of the attic is shown in Figure 1b. 
Postprint: Das P, Van Gelder L, Janssen H, Roels S. 2017. Designing uncertain optimization schemes for the economic assessment of stock energy-efficiency measures, Journal of Building Performance Simulation, 10:3-16. doi: 10.1080/19401493.2015.1099054

The risk of mould growth is determined from a mathematical model formulated from previous regression analyses for mould growth on sapwood of pine and spruce (Hukka and Viitanen 1999). The mould was measured applying an existing standard index $(M)$ based on the visual appearance of the surface, taking any value between 0 and 6 where:

0 no growth

1 some growth only detected with microscopy

2 moderate growth detected with microscopy (coverage more than 10\%)

3 some growth detected visually

4 visually detected coverage more than $10 \%$

5 visually detected coverage more than $50 \%$

6 visually detected coverage $100 \%$

The rate of change in $M$ is given by a differential equation that is a function of the relative humidity and temperature. It is subject to a critical relative humidity that depends on the temperature below which there is no mould growth. It is also bounded by a maximum mould coverage that depends on the temperature and humidity conditions. The form also varies for the initiation of mould growth $(M<1)$ versus the first visual appearance of mould growth $(1<M<3)$. In fluctuating conditions, there is assumed to be a delay in mould growth, which is a function of the time passed from the beginning of the dry period.

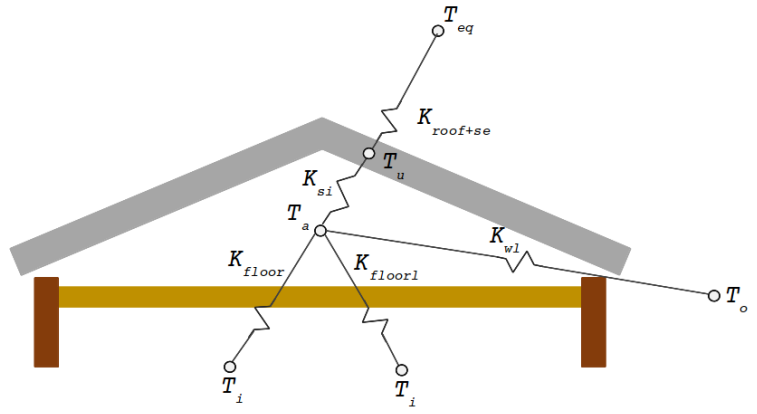

(a)

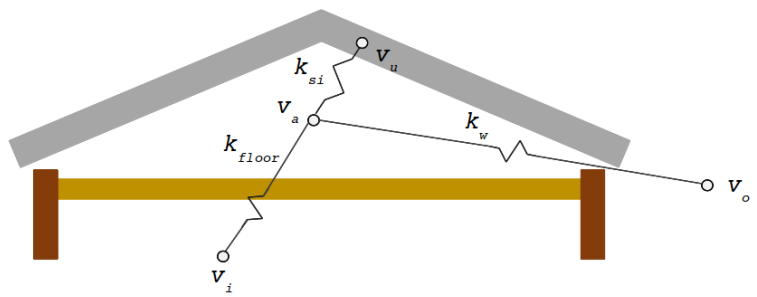

(b)

Figure 1.: The (a) thermal and (b) moisture networks in the attic.

The cold attic model has $n_{\text {pars }}=15$ input parameters, shown in Table 1 along with parameters describing how they may vary across a typical Swedish stock of dwellings. The outputs of the model are the yearly peak mould growth index for the wooden underlay $(P M G,-)$, and the heat loss through the ceiling accumulated over the heating season $(C H L, \mathrm{kWh})$ for a period of 10 years.

\subsection{Retrofitting scenarios}

Several energy efficiency measures are considered as a method of reducing energy use in the casestudy housing stock:

- Increasing the insulation level of the attic floor, i.e. reducing the U-value to a target value of

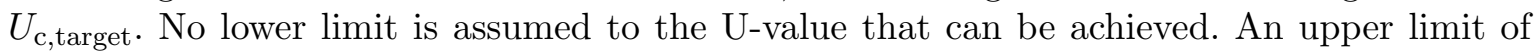
2.3 is assumed, equivalent to the current mean of the case-study stock.

- Increasing the airtightness of the attic floor, i.e. reducing the effective leakage area of the attic floor to a target value of $A_{\mathrm{c}, \text { target }}$. The lowest achievable effective leakage area is considered to be $5 \times 10^{-8} \mathrm{~m}^{2} / \mathrm{m}^{2}$. The upper limit is set to $6.3 \times 10^{-5} \mathrm{~m}^{2} / \mathrm{m}^{2}$, the current mean of the case-study stock.

- Sealing the ventilation gaps at the eaves, i.e. reducing the venting area per metre eave to a target value of $A_{\mathrm{e}, \text { target }}$. The airtightness of the eaves has a lower limit of $2.5 \times 10^{-5} \mathrm{~m}^{2} / \mathrm{m}$ 
Postprint: Das P, Van Gelder L, Janssen H, Roels S. 2017. Designing uncertain optimization schemes for the economic assessment of stock energy-efficiency measures, Journal of Building Performance Simulation, 10:3-16. doi: 10.1080/19401493.2015.1099054

and an upper limit of $0.023 \mathrm{~m}^{2} / \mathrm{m}$, again the current mean of the case-study stock.

Table 1.: Input parameters and their associated probability distributions for the case-study stock. ' $\mathrm{U}$ ' signifies a continuous uniform distribution ( $p_{1}$ is the minimum and $p_{2}$ is the maximum) except for 'Initial year' for which it corresponds to a discrete uniform distribution. ' $\mathrm{N}$ ' represents a normal distribution ( $p_{1}$ is the mean and $p_{2}$ is the standard deviation).

\begin{tabular}{llllc}
\hline Input & Symbol & $p_{1}$ & $p_{2}$ & Distribution \\
\hline \hline Building height $(\mathrm{m})$ & $H_{\mathrm{b}}$ & 4 & 8 & $\mathrm{U}$ \\
Roof and ceiling area $\left(\mathrm{m}^{2}\right)$ & $A$ & 50 & 200 & $\mathrm{U}$ \\
Roof eave angle $\left({ }^{\circ}\right)$ & $\theta_{\mathrm{e}}$ & 0 & 180 & $\mathrm{U}$ \\
Roof eave effective leakage area per metre $\left(\mathrm{m}^{2} / \mathrm{m}\right)$ & $A_{\mathrm{e}}$ & 0.01 & 0.05 & $\mathrm{U}$ \\
Roof eave and building length $(\mathrm{m})$ & $\mathrm{L}$ & 7 & 20 & $\mathrm{U}$ \\
Thickness of wooden underlay $(\mathrm{m})$ & $d_{\mathrm{u}}$ & 0.01 & 0.02 & $\mathrm{U}$ \\
Vapour diffusion coefficient of wooden underlay $\left(\mathrm{m}^{2} / \mathrm{s}\right)$ & $\delta_{\mathrm{u}}$ & $1 \times 10^{-6}$ & $2 \times 10^{-7}$ & $\mathrm{~N}$ \\
Initial relative humidity at wooden underlay centre $(\%)$ & $\phi_{\mathrm{u}, \mathrm{i}}$ & 0.5 & 0.9 & $\mathrm{U}$ \\
Thermal conductivity of wooden underlay $(\mathrm{W} /(\mathrm{mK}))$ & $\lambda_{\mathrm{u}}$ & 0.13 & 0.02 & $\mathrm{~N}$ \\
Thermal resistance of roof insulation $\left(\mathrm{m}^{2} \mathrm{~K} / \mathrm{W}\right)$ & $R_{\mathrm{r}}$ & 0 & 1 & $\mathrm{U}$ \\
Ceiling effective leakage area per square metre $\left(\mathrm{m}^{2} / \mathrm{m}^{2}\right)$ & $A_{\mathrm{c}}$ & $10^{-5}$ & $10^{-4}$ & $\mathrm{U}$ \\
Ceiling U-value $\left(\mathrm{W} /\left(\mathrm{m}^{2} \mathrm{~K}\right)\right)$ & $U_{\mathrm{f}}$ & 1 & 3 & $\mathrm{U}$ \\
Indoor area temperature $\left({ }^{\circ} \mathrm{C}\right)$ & $T_{\mathrm{i}}$ & 20 & 1.5 & $\mathrm{~N}$ \\
Humidity of indoor moisture supply $\left(\mathrm{kg} / \mathrm{m}^{3}\right)$ & $v_{\mathrm{ims}}$ & 0.005 & 0.002 & $\mathrm{~N}$ \\
Initial year $(-)$ & $y_{0}$ & 1 & 30 & $\mathrm{U}$ \\
\hline
\end{tabular}

Each design option is a combination of one or more of these energy efficiency measures. The costs of each of the energy efficiency measures is shown in Table 2. The data is based on expert knowledge available in the IEA EBC Annex 55-project, though it is by no means the aim to be as precise as possible, but rather to gather realistic data that could be used to check the methodology. As can be seen in Table 2, a one-off repair cost $\left(€ 58 / \mathrm{m}^{2}\right)$ in the case that $P M G$ exceeds a value of five at least once within the 10 years since the initial retrofitting has also been taken into account. There are uncertainties with the installation of each energy efficiency measure, also shown in Table 2. Therefore the actual new dwelling characteristics will vary from the target dwelling characteristics.

Table 2.: Energy efficiency measures, uncertainty in their implementation, and their costs.

\begin{tabular}{|c|c|c|c|c|c|}
\hline Energy efficiency measure & Old value & Target value & Actual value & Cost & \\
\hline Ceiling U-value & $U_{\mathrm{c}, \mathrm{old}}$ & $U_{\mathrm{c}, \text { target }}$ & $U_{\mathrm{c}, \text { new }}=N\left(U_{\mathrm{c}, \text { target }}, 0.1 U_{\mathrm{c}, \text { target }}\right)$ & $8.0+1.2\left(\frac{1}{U_{\mathrm{c}, \text { new }}}-\frac{1}{U_{\mathrm{c}, \text { old }}}\right)$ & $€ / \mathrm{m}^{2}$ \\
\hline Ceiling airtightness & $A_{\mathrm{c}, \mathrm{old}}$ & $A_{\mathrm{c}, \text { target }}$ & $A_{\mathrm{c}, \text { new }}=N\left(A_{\mathrm{c}, \text { target }}, 0.2 A_{\mathrm{c}, \text { target }}\right)$ & $5.0+\frac{3 \times 10^{-7}}{A_{\mathrm{c}, \text { new }}} € / \mathrm{m}^{2}$ & \\
\hline Roof eave airtightness & $A_{\mathrm{e}, \mathrm{old}}$ & $A_{\mathrm{e}, \text { target }}$ & $A_{\mathrm{e}, \text { new }}=N\left(A_{\mathrm{e}, \text { target }}, 0.4 A_{\mathrm{e}, \text { target }}\right)$ & $12.0+\frac{3 \times 10^{-4}}{A_{\mathrm{e}, \text { new }}} € / \mathrm{m}$ & \\
\hline
\end{tabular}

Each design option is assessed over the period of 10 years $(y)$ by an economic criterion that depends in some way on the retrofitting costs, energy savings, and in general on whether mould damage occurs. An energy price per $\mathrm{kWh}, P_{E}$, of $€ 0.1$ is assumed and Table 3 shows the distributions assumed for the inflation-corrected discount rate and annual increment in energy cost, reflecting the uncertainty in future economic scenarios.

Table 3.: Uncertainty in future economic scenarios.

\begin{tabular}{lll}
\hline Economic variable & Symbol & Distribution \\
\hline Inflation-corrected discount rate & $a$ & $N(0.07,0.015)$ \\
Inflation-corrected annual increment in energy cost & $r_{E}$ & $N(0.065,0.0175)$ \\
\hline
\end{tabular}


Postprint: Das P, Van Gelder L, Janssen H, Roels S. 2017. Designing uncertain optimization schemes for the economic assessment of stock energy-efficiency measures, Journal of Building Performance Simulation, 10:3-16.

doi: 10.1080/19401493.2015.1099054

\section{4. $\quad$ Metamodel of cold attic model}

The cold attic model takes about 10 minutes to run one simulation on a quad-core Intel-5 processor, and therefore running at least a few hundred to reflect the stock, weather, and economic variability for each design option, and then at least a few hundred design options to explore the optimal scenario, will be a computationally demanding task. Therefore a neural network is constructed to reduce the total simulation time; more details regarding the construction of a neural network can be found in previous work (Das et al. 2014b; Van Gelder et al. 2014a). The inputs to the neural network are the same as those for the cold attic model. The outputs however are a diagnostic for the incidence of mould damage in the attic during the 10 years and the values of $C H L$ for each of the 10 years (i.e., 11 outputs altogether). The maintenance cost resulting from any mould damage is only dependent on whether $P M G \geq 5$ at least once in the 10 years. Therefore the mould diagnostic can be just one parameter (either 0 or 1 ) representing whether a maintenance cost is required, or not. A sample of size 500 is constructed using simple random sampling for the training of the metamodel, assuming input parameter distributions given in Table 1, except for the following parameters:

- $U_{\mathrm{c}} \in[0.0,3.0]$ Uniform

- $A_{\mathrm{c}} \in\left[5.0 \times 10^{-8}, 1.0 \times 10^{-4}\right]$ Uniform on a logarithmic scale

- $A_{\mathrm{e}} \in\left[2.5 \times 10^{-5}, 0.05\right]$ Uniform on a logarithmic scale

These ranges are chosen to cover the full range of dwelling characteristics of the old stock and likely new stock after retrofitting. $A_{\mathrm{c}, \text { target }}$ and $A_{\mathrm{e}, \text { target }}$ are sampled uniformly on a logarithmic scale to ensure that the metamodel mimics the behaviour at very small values of these dwelling characteristics. Various multi-layer neural network architectures are explored; the first for the mould diagnostic, which is binary and therefore treated as a classification problem; a second for the $C H L$ outputs for each of the 10 years. The following variations are considered ${ }^{2}$ :

(1) Neural network architecture:

- Network type:

o feedforwardnet ( $f f n$ ) has tan-sigmoidal activiation functions except for the final layer which is linear. Only adjacent layers are connected.

- cascasdeforwardnet ( $\mathrm{cfn}$ ) is like $\mathrm{ffn}$ but also enables connections between nonadjacent layers.

- patternnnet (pn) is similar to $\mathrm{ffn}$ but has also a tan-sigmoidal or log-sigmoidal final layer, and is therefore suitable for classification problems.

- Number of hidden layers.

- Number of neurons per layer (assumed to be the same per layer).

(2) Training algorithm:

- Levenberg-Marquardt algorithm (trainlm).

- Bayesian regularization algorithm (trainbr).

- Scaled conjugate gradient backpropagation (trainscg). Suitable for pattern recognition networks.

- Resilient backpropagation (trainrp). Suitable for pattern recognition networks.

(3) Method for division of design sample into training, validation, and testing samples:

- Random division (dividerand).

- Division by specified indices (divideind).

For both metamodels, dividerand is used to find the best division of the sample for different random seeds and the indices are then saved. The division method is then switched to divideind to compare between neural network architectures and training algorithms by looking at the mean squared error $(M S E)$ of the test sample. For the mould diagnostic metamodel, the pn architecture

\footnotetext{
${ }^{2}$ The use of the typewriter font denotes a MATLAB function.
} 
Postprint: Das P, Van Gelder L, Janssen H, Roels S. 2017. Designing uncertain optimization schemes for the economic assessment of stock energy-efficiency measures, Journal of Building Performance Simulation, 10:3-16. doi: 10.1080/19401493.2015.1099054

is explored, with between one and three hidden layers, between two and 20 neurons per layer, and using the trainscg and trainrp training algorithms. For the $C H L$ metamodel, the $\mathrm{ffn}$ and $\mathrm{cfn}$ architectures are explored, with between one and two hidden layers, between two and 15 neurons per layer, and using the trainlm and trainbr training algorithms. Once the best architecture and training algorithm combination is found, the random seed is again varied to determine the best possible neural network. If the model is still not satisfactory, the whole procedure can be repeated several times. The preferred architecture for the mould diagnostic is the pn architecture with two layers, each with 12 neurons and the trainscg training algorithm. Rounding up the metamodel outputs to 0 (if $<0.5$ ) or 1 (if $\geq 0.5$ ), gives a misclassification rate of $10 \%$ for the testing sample. To determine the best metamodel for the mould diagnostic requires a few loops of the procedure outlined above. The preferred architecture for the $10 \mathrm{CHL}$ outputs is the $\mathrm{ffn}$ architecture with one layer, each with five neurons and the trainbr training algorithm. Only one loop of the procedure described above is required as the metamodel fits these outputs very well in general. Regression plots comparing the $C H L$ targets to the metamodel outputs are shown in Figure 2.
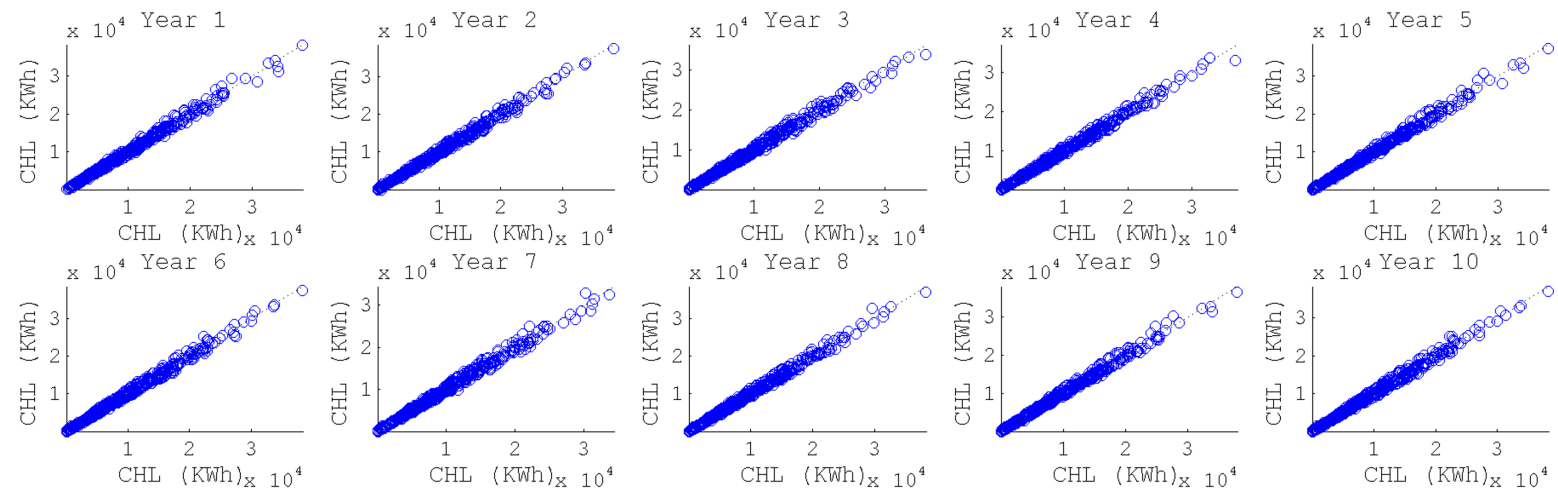

Figure 2.: Regression between the metamodel and cold attic model predictions of annual cumulated heat losses $(C H L)$ for each of the 10 years since the initial retrofitting. The $R^{2}$ in each case exceeds 0.995 .

\section{Uncertain optimization methodology}

In this section, we discuss the various options explored in this paper in the design of an uncertain optimization framework. This includes making choices about the criterion used to assess each design option and the extraction of a deterministic objective function from a criterion distribution. A method for uncertainty propagation needs to be selected in the evaluation of the assessment criterion that incorporates the impact of variabilities in the dwelling characteristics across the stock, uncertainties in the retrofitting process, and uncertainties in the future economic scenario. Finally, a method needs to be selected for exploring the parameter space of design options.

\subsection{Economic criterion}

Four different economic criteria are investigated for assessing the performance of a particular design option, covering varying treatments of maintenance costs, changes in energy prices, discount rates, and absolute returns compared to fractional and annulized returns. Several definitions of each of these criteria exist in the literature; the choices made in this work are detailed below. 
Postprint: Das P, Van Gelder L, Janssen H, Roels S. 2017. Designing uncertain optimization schemes for the economic assessment of stock energy-efficiency measures, Journal of Building Performance Simulation, 10:3-16.

\subsubsection{Payback period (PB)}

An investment that leads to a cash flow in the future can be assessed by the payback period $(P B)$, which is a measure of how long it takes for the initial investment to be paid back, usually without consideration of any maintenance costs, a discount rate, or a change in energy costs:

$$
\sum_{l=1}^{P B} \frac{\Delta K_{\mathrm{E}, l}}{I_{0}}=1
$$

where $I_{0}$ is the initial cost of the design option and $\Delta K_{E, l}$ is the change in energy costs in year $l$ :

$$
\Delta K_{\mathrm{E}, l}=P_{E} \Delta E_{\text {use,heating }, l}
$$

where $\Delta E_{\text {use,heating, } l}$ is the difference in $C H L$ between the original and retrofitted dwelling in year $l$.

\subsubsection{Net Present Value (NPV)}

The present value of future cash flows is discounted back to the time of the investment to compare different investment alternatives, taking into account any maintenance costs, changes in energy prices, and a discount rate. The total Net Present Value $(N P V)$ after a chosen number of years, $y$, since the retrofitting is defined as:

$$
N P V=-I_{0}-I_{\mathrm{M}}+\sum_{l=1}^{y} \frac{\Delta K_{\mathrm{E}, l}\left(1+r_{\mathrm{E}}\right)^{l}}{(1+a)^{l}}
$$

where $I_{0}$ and $\Delta K_{\mathrm{E}, l}$ are defined above and $I_{\mathrm{M}}$ is the maintenance cost. $N P V$ is therefore a measure of the absolute return after a fixed time period.

\subsubsection{Return on Investment (ROI)}

In reality, different retrofitting scenarios will incur different initial investment costs. The Return on Investment $(R O I)$ is a measure of the efficiency of the investment defined as the ratio of $N P V$ and the sum of the initial investment and any maintenance costs:

$$
R O I=\frac{N P V}{I_{0}+I_{\mathrm{M}}}
$$

where $N P V, I_{0}$, and $I_{\mathrm{M}}$ are defined above. Therefore it is a measure of the fractional return after a fixed time period.

\subsubsection{Internal rate of return (IRR)}

The Internal Rate of Return $(I R R)$ is the inflation-corrected discount rate, $a$, for the design option where the $N P V$ equals 0 , i.e.

$$
N P V=0 \Rightarrow I_{0}+I_{\mathrm{M}}=\sum_{l=1}^{y} \frac{\Delta K_{\mathrm{E}, l}\left(1+r_{\mathrm{E}}\right)^{l}}{\left(1+a_{I R R}\right)^{l}}
$$

where $a_{I R R}$ is calculated by an iterative process. It is therefore again a measure of fractional return, but it is also annualized, enabling projects of different lifespans to be compared. 
Postprint: Das P, Van Gelder L, Janssen H, Roels S. 2017. Designing uncertain optimization schemes for the economic assessment of stock energy-efficiency measures, Journal of Building Performance Simulation, 10:3-16.

\subsection{Objective function}

A deterministic objective function needs to be extracted from the probabilistic economic criterion. We first consider a weighted sum of the expected value and the standard deviation, thus enabling the degree of risk to be considered as well as the mean impact:

$$
f(x)=-\theta_{1} E(x)+\theta_{2} \sigma(x),-1 \leq \theta_{2} \leq 1, \theta_{1}+\left|\theta_{2}\right|=1
$$

Risk-neutral decision makers are not concerned with risk and therefore would choose $\theta_{2}=0$. This minimizes the negative of the expected value of the economic criterion (denoted by $x$ ), thus allowing the expected value to be maximized. Risk-taking decision makers are willing to take a risk that the probability of a poor performance is increased if it also means that the probability of a very good performance is increased. In this case the weighted-sum of the negative of the expected value and the dispersion of the economic criterion distribution is minimized, and therefore a negative value would be selected for $\theta_{2}$. Risk-averse decision makers are not willing to take a risk on their investment and would like to be assured of little variation in the result for a stock, and therefore a positive value would be selected for $\theta_{2}$.

The optimization can therefore be formally stated as:

$$
\min _{x \in \Re^{n}} f(x)
$$

where we note that the objective function is not everywhere differentiable due to the binary nature of the maintenance costs included in some of the economic criteria.

We also consider an alternative objective function that could be used by a risk-averse decision maker where the probability $P(N P V)<0$ is directly minimized, because it may be that the decision maker is more concerned with ensuring that there is no negative result.

\subsection{Sampling input variations}

The economic criterion distribution for each design option is calculated for a Latin Hypercube design sample of dwelling characteristics, year, and future economic scenarios. The usual Latin Hypercube method is replaced with one using mini-Latin Hypercube samples of size 20 (Janssen 2013). The mean and standard deviation of the output economic criterion distribution is calculated for each mini-sample and this is repeated until the internal standard deviation of both the mean and standard deviation of the output economic criterion distribution changes less than $0.2 \%$. This value is used as a balance between computational time and accuracy. A higher value lacks repeatability, while a smaller value becomes more computationally demanding.

\subsection{Generating design options}

There are several different ways of generating design options. Two different combinations for generating the design options are detailed here: a hybrid genetic algorithm and a multi-layered sampling scheme. Rather than saving the whole economic criterion distribution for each generated design option, only the parameters of the design option, the objective function, the mean and standard deviation of the economic criterion distribution used in the objective function, and the mean, standard deviation, and probability of $N P V$ being less than zero are saved for any further analysis. Saving NPV moments even in the case where the objective function depends on alternate economic criteria enables a comparison to be made between optimal design options using a common variable. 
Postprint: Das P, Van Gelder L, Janssen H, Roels S. 2017. Designing uncertain optimization schemes for the economic assessment of stock energy-efficiency measures, Journal of Building Performance Simulation, 10:3-16. doi: 10.1080/19401493.2015.1099054

\subsubsection{Hybrid genetic algorithm}

This method uses the objective functions associated with previously sampled design options to help select the next design option, therefore only investigating part of the whole design parameter space in detail. The method has the advantage of being able to deal with non-differentiable functions, which may be the case with our objective function. The genetic algorithm initially randomly generates a population of chromosomes, each corresponding to a design option. The objective function is evaluated for each chromosome in the population. The new population for the next iteration is formed by randomly selecting multiple chromosomes from the current population, depending on their associated objective function, and recombining them with possible random mutations. The proportion of chromosomes in the current generation that will survive into the next generation is governed by the crossover fraction. The genetic algorithm is specified so that it stops once either a maximum number of generations has been reached or once the mean relative change in the objective function value over a specified number of generations is less than or equal to some specified level of accuracy. The genetic algorithm is used to find the region containing the solution, and then the much more computationally efficient gradient-descent algorithm finds the precise solution. We expect that the objective function is differentiable close to the optimum as inclusion/exclusion of a maintenance cost should move the objective function in or out of that region. A population size of 20 is used for the genetic algorithm, a crossover fraction of 0.6 , and a maximum number of 100 generations. The genetic algorithm exits once the optimal design option changes less than $0.2 \%$, the same accuracy used for the Latin Hypercube sampling convergence.

\subsubsection{Multi-layered sampling}

The multi-layered sampling scheme employed here is a modified version of that presented in Van Gelder et al. (2014b), with the three-layered scheme condensed into a two-layered scheme here. The first layer (Figure 3) represents all potential design options and the second layer is the sampling of input variations discussed above in Section 3.3. In Van Gelder et al. (2014b), the controllable variations linked with the dwelling characteristics and uncontrollable variations linked with the future economic scenario are sampled separately, to enable an assessment to be made of how the overall optimal design option compares to the optimal design option in varying economic situations. The Latin Hypercube design is used here to create a sample of 500 design options.

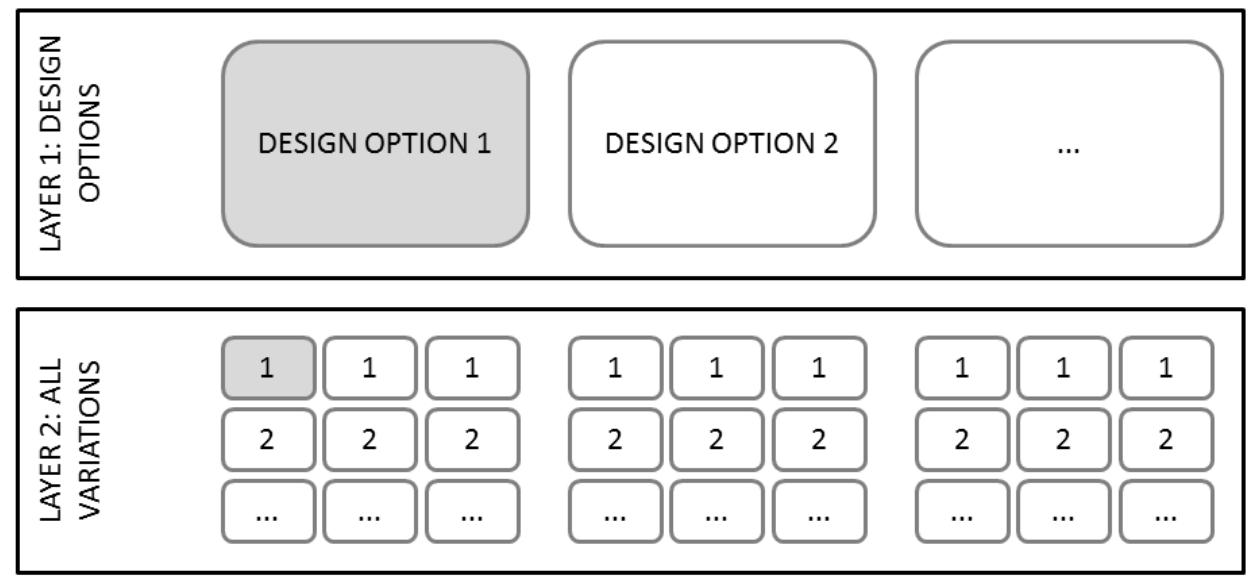

Figure 3.: Two-layered sampling design, separating design options (top layer) from both uncontrollable and controllable variations (bottom layer). 
Postprint: Das P, Van Gelder L, Janssen H, Roels S. 2017. Designing uncertain optimization schemes for the economic assessment of stock energy-efficiency measures, Journal of Building Performance Simulation, 10:3-16. doi: 10.1080/19401493.2015.1099054

\section{Results}

Here the computational performance of the metamodel, the impact of the choice of economic criterion, objective function formulation, and scheme for generating design options on the derived optimal design option is described.

\subsection{Computational performance of the metamodel}

An important assessment of the metamodel is to analyse its computational performance. As stated earlier, one evaluation of the cold attic model takes 697s (just over 10 minutes) on a quad-core Intel-5 processor. Therefore the time to carry out the 500 simulations required for training the neural network is almost 100 hours. The time required for training each neural network is about two hours, amounting to about four hours in the case of training both neural networks. The minimum and maximum run times for the optimization with the metamodel are one hour for the base-case uncertain optimzation scheme and about 19 hours for the multi-layered uncertain optimization scheme, though the latter also contains all the Pareto-optimal solutions. If we had used the cold attic model rather than the metamodel in the case of the multi-layered uncertain optimization scheme, we would have required approximately $500 \times 200$ evaluations of the cold attic model (500 for the number of design options, and around 200 for the Monte Carlo stock for each design option), amounting to almost 20,000 hours, which would have been infeasible. Even in the case of the base-case uncertain optimization scheme, around 1000 hours would have been required.

\subsection{Base-case uncertain optimization scheme}

The base-case uncertain optimization scheme $\left(S_{\mathrm{bc}}\right)$ uses the $N P V$ economic criterion, an equal weight between the expected value and standard deviation of the $N P V$ distribution (i.e. $\theta_{2}=0.5$ in Equation (6)), and the hybrid genetic algorithm for generating potential design options. For this particular optimization run, convergence occurred after 206 generations of the genetic algorithm. The optimal design option (Table 4) prefers a significant reduction in the U-value of the attic floor from the current stock mean of $2.3 \mathrm{~W} / \mathrm{m}^{2} / \mathrm{K}$, a similar value for the ceiling effective leakage area to the current stock mean of $6.3 \times 10^{-5} \mathrm{~m}^{2} / \mathrm{m}^{2}$ and a roof eave effective leakage area per metre that is significantly lower than the current stock mean of $0.023 \mathrm{~m}^{2} / \mathrm{m}$.

The cumulative distribution of $N P V$ that would be expected in a stock of dwellings undergoing such a retrofitting scenario is illustrated in Figure 4. The optimal design option would result in a mean $N P V$ of $€ 151510$ years after the installation of the energy efficiency measures, but there would be a significant dispersion in the results across a stock. The distribution also shows that we would expect $37 \%$ of dwellings to achieve only a negative $N P V$ as a result of a lack of significant energy savings compared to the sum of retrofitting and maintenance costs.

\subsection{Impact of the economic criterion}

The impact of varying the economic criterion in the base-case scheme $\left(S_{\mathrm{bc}, E C}\right)$ on the optimal design option is summarised in Table 4. All four economic criteria prefer an attic floor U-value significantly lower than the current stock mean. The $P B$ and $I R R$ criteria prefer an attic floor effective leakage area similar to the current mean, while the $R O I$ criterion prefers a much lower value, though within the range found in the stock. The preferred roof eave effective leakage area appears to vary considerably between criteria.

Figure 5 shows the cumulative economic criterion distributions for the optimal design options derived for each economic criterion. The optimal design option using the $P B$ criterion finds an expected period of 1.68 years with a standard deviation of 0.88 years. The standard deviation is less than the expected value for this criterion. The optimal design option using the ROI criterion 
Postprint: Das P, Van Gelder L, Janssen H, Roels S. 2017. Designing uncertain optimization schemes for the economic assessment of stock energy-efficiency measures, Journal of Building Performance Simulation, 10:3-16. doi: 10.1080/19401493.2015.1099054

Table 4.: Optimal design option, expected value, standard deviation, probability of $N P V$ being less than 0 and (where different) the expected value and standard deviation of the economic criterion distribution used in the objective function. These are given for the base-case uncertain optimization scheme, and variants on the choice of economic criterion and method of generating design options. For comparison, the current stock mean U-value of the attic floor is $2.3 \mathrm{~W} / \mathrm{m}^{2} / \mathrm{K}$, effective leakage area in the attic floor is $6.3 \times 10^{-5} \mathrm{~m}^{2} / \mathrm{m}^{2}$, and effective leakage area in the attic eaves is $0.023 \mathrm{~m}^{2} / \mathrm{m}$.

\begin{tabular}{|c|c|c|c|c|c|c|c|c|}
\hline Variant & $\begin{array}{l}U_{\mathrm{c}, \text { target }} \\
\left(\mathrm{W} / \mathrm{m}^{2} / \mathrm{K}\right)\end{array}$ & $\begin{array}{l}A_{\mathrm{c}, \text { target }} \\
\left(\mathrm{m}^{2} / \mathrm{m}^{2}\right)\end{array}$ & $\begin{array}{l}A_{\mathrm{e}, \text { target }} \\
\left(\mathrm{m}^{2} / \mathrm{m}\right)\end{array}$ & $E(N P V)$ & $\sigma(N P V)$ & $P(N P V<0)$ & $E(E C)$ & $\sigma(E C)$ \\
\hline$\overline{S_{\mathrm{bc}}}$ & 0.17 & $5.66 \times 10^{-5}$ & 0.0024 & $€ 1515$ & $€ 4200$ & 0.37 & - & - \\
\hline$S_{\mathrm{bc}, P B}$ & 0.25 & $6.22 \times 10^{-5}$ & 0.0011 & $€ 1091$ & $€ 4030$ & 0.40 & $2.49 \mathrm{yrs}$ & $0.88 \mathrm{yrs}$ \\
\hline$S_{\mathrm{bc}, R O I}$ & 0.13 & $1.16 \times 10^{-5}$ & 0.010 & $€ 1411$ & $€ 4259$ & 0.38 & 0.16 & 0.40 \\
\hline$S_{\mathrm{bc}, I R R}$ & 0.14 & $6.07 \times 10^{-5}$ & 0.00058 & $€ 1598$ & $€ 4226$ & 0.37 & 0.096 & 0.072 \\
\hline$S_{\mathrm{bc}, P(N P V<0)}$ & 0.14 & $6.30 \times 10^{-5}$ & 0.00078 & $€ 1681$ & $€ 4378$ & 0.37 & - & - \\
\hline$S_{\mathrm{ml}}$ & 0.14 & $4.65 \times 10^{-5}$ & 0.0023 & $€ 1555$ & $€ 4232$ & 0.37 & - & - \\
\hline$S_{\mathrm{ml}, P(N P V<0)}$ & 0.14 & $4.65 \times 10^{-5}$ & 0.0023 & $€ 1555$ & $€ 4232$ & 0.37 & - & - \\
\hline
\end{tabular}

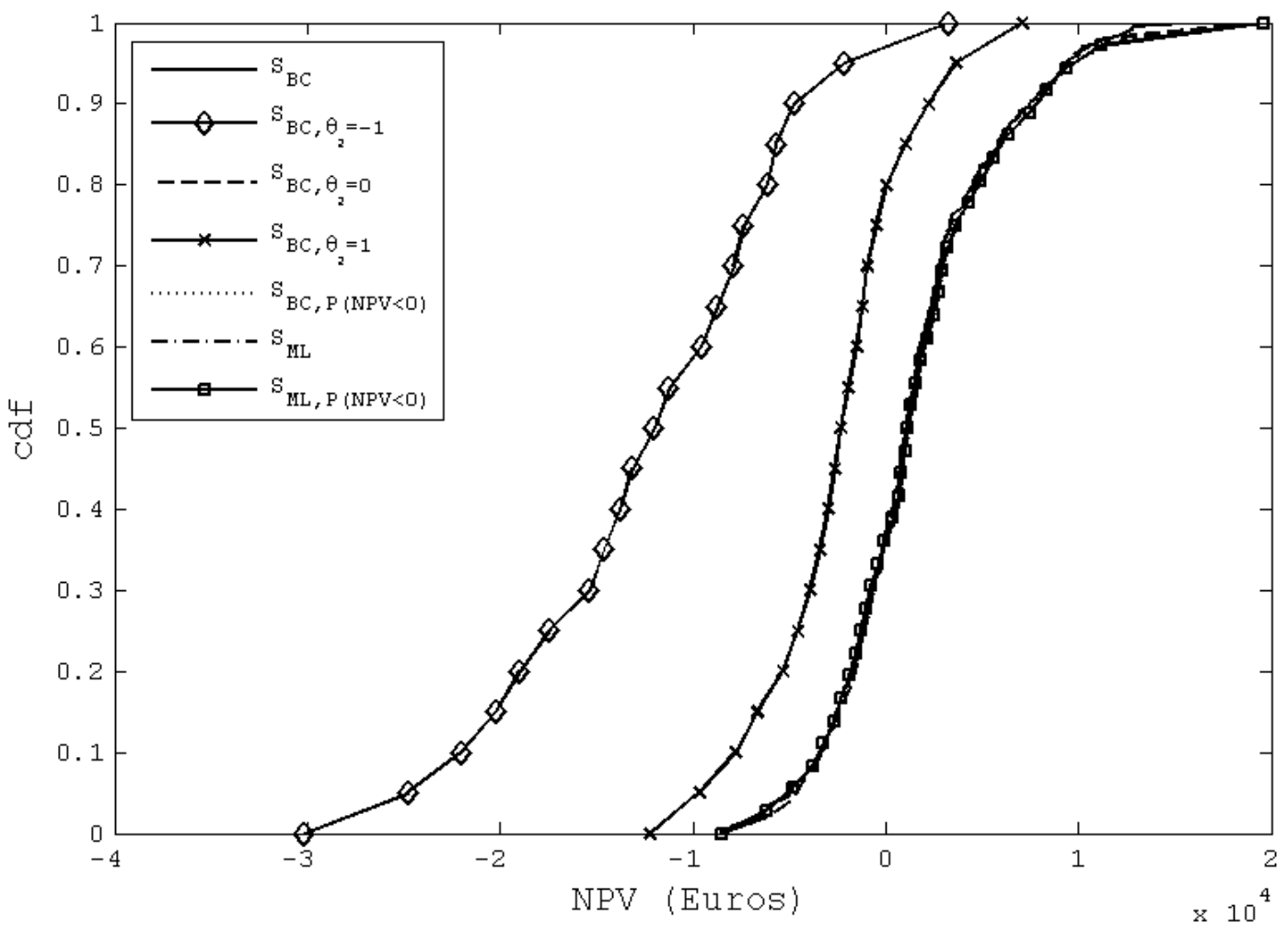

Figure 4.: NPV cumulative distribution function (cdf) associated with the optimal design options for several variants in the optimization scheme.

finds an expected return of $16 \%$, but with a high dispersion across the stock of $40 \%$. As with the $N P V$ criterion, $37 \%$ of the stock is expected to get negative returns. The $I R R$ criterion finds an expected rate of return of almost $10 \%$ with a dispersion of about $7 \%$. About $10 \%$ of the stock would expect to have a negative $I R R$, while about $10 \%$ of the stock will have an $I R R$ exceeding $20 \%$.

Table 4 and Figure 4 also describe the $N P V$ distributions that would result from the optimal design options found using each of the economic criteria, enabling a more direct comparison between them. All four $N P V$ distributions are remarkably similar in profile. The only profile that can be 
Postprint: Das P, Van Gelder L, Janssen H, Roels S. 2017. Designing uncertain optimization schemes for the economic assessment of stock energy-efficiency measures, Journal of Building Performance Simulation, 10:3-16. doi: 10.1080/19401493.2015.1099054

distinguished is that where $P B$ is used as the economic criterion. $P(N P V<0)$ is 0.40 rather than 0.37 , and the dispersion is smaller but at the expense of a lower mean.
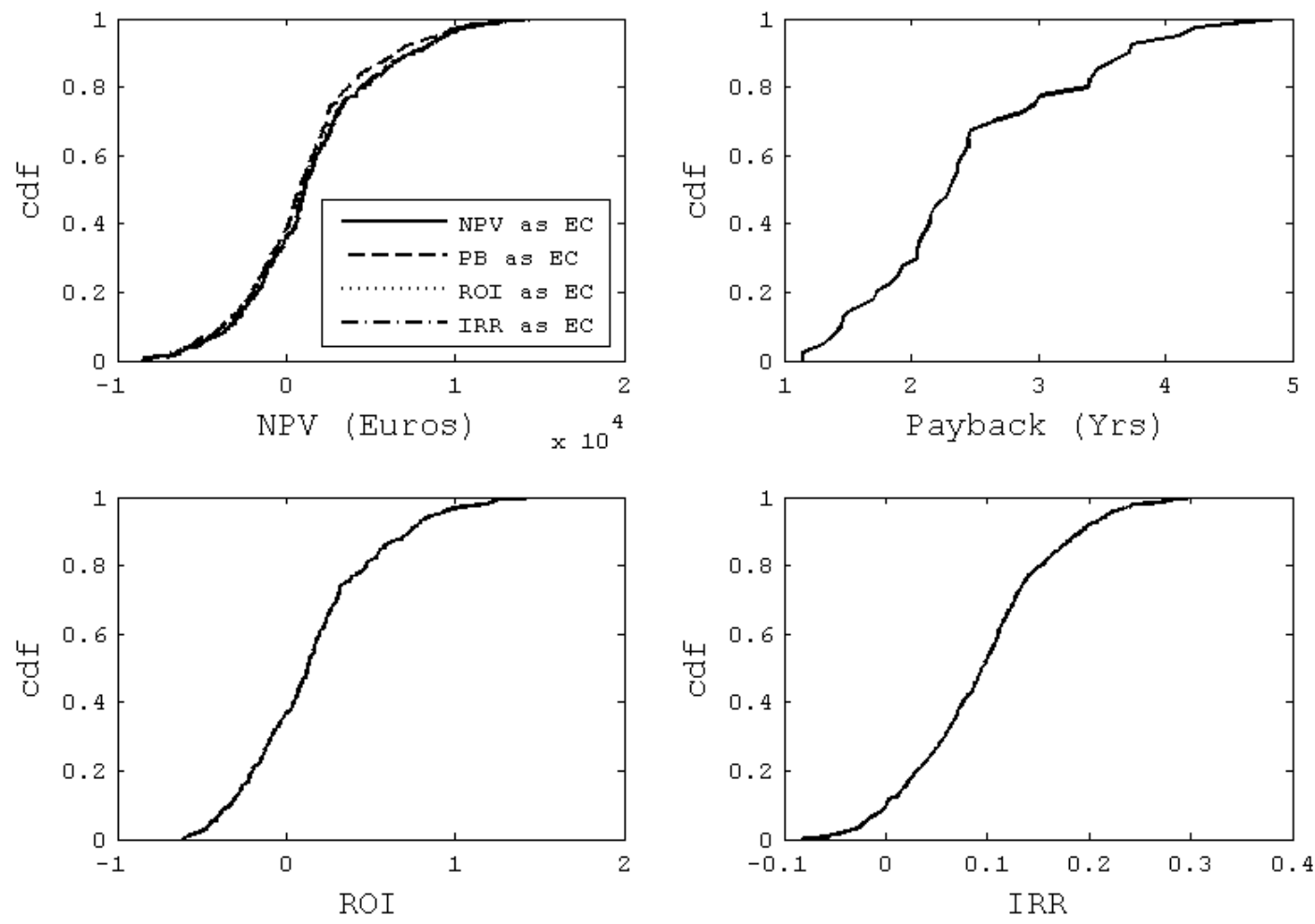

Figure 5.: Economic criterion cumulative distribution function (cdf) for optimal design option for the various choices of the economic criterion. The top left plot also shows the $N P V$ distributions corresponding to the optimal design options found using the other economic criteria in the objective function.

\subsection{Impact of the objective function formulation}

The impact of the balance between the expected value and standard deviation of $N P V$ in the base-case scheme is shown in Figure 6 . As the weight on the standard deviation of the $N P V$ distribution in the objective function, $\theta_{2}$, increases from -1 to 1 , the optimal target $U_{\mathrm{c}, \text { target }}$ starts at 0 when only standard deviation is considered in a risk-taking sense, and then increases slightly between $0.12-0.22$ before jumping to 0.79 when only the standard deviation is considered in a riskaverse sense. The leakage area in the attic floor stays close to the current stock mean, except when $\theta_{2}=-1$, which results in a value of zero. The leakage area in the eaves decreases rapidly from the stock mean at first as $\theta_{2}$ increases, before levelling off at a value about an order of magnitude smaller than the stock mean.

The expected $N P V$ is relatively constant around $€ 1600$ except in the cases where only the standard deviation is considered in the objective function, in which case the expected $N P V$ falls to negative values. The standard deviation of the $N P V$ distribution falls as $\theta_{2}$ increases, steeply at first and then more steadily. The impact of $\theta_{2}$ on the shape of the $N P V$ distribution can be seen in Figure 4. The distribution for $\theta_{2}=0$ is very similar to that for $\theta_{2}=0.5$. The distribution for $\theta_{2}=-1$ however is much wider and the distribution for $\theta_{2}=1$ is narrower, as expected. Trying to reduce the dispersion in the results leads to a higher proportion with negative $N P V$. 
Postprint: Das P, Van Gelder L, Janssen H, Roels S. 2017. Designing uncertain optimization schemes for the economic assessment of stock energy-efficiency measures, Journal of Building Performance Simulation, 10:3-16. doi: 10.1080/19401493.2015.1099054

However, encouraging a high dispersion with the aim of increasing the probability of a very high $N P V$ also results in the whole stock likely to only achieve a negative $N P V$. In these cases, the combination of investment costs and maintenance costs must exceed the energy savings on average. The optimal design option obtained using $P(N P V<0)$ as the objective function in the base-case

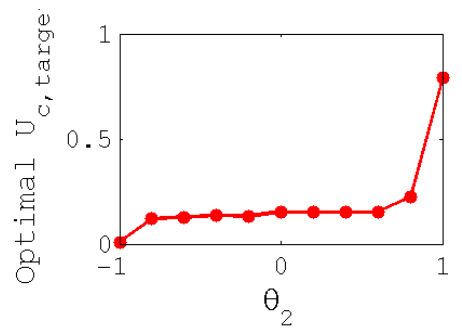

(a)

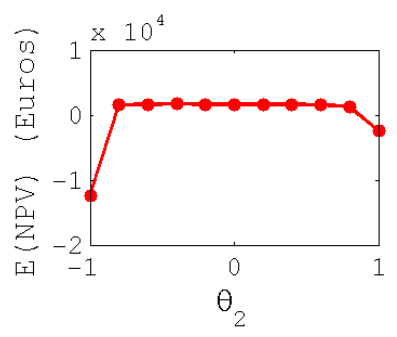

(d)

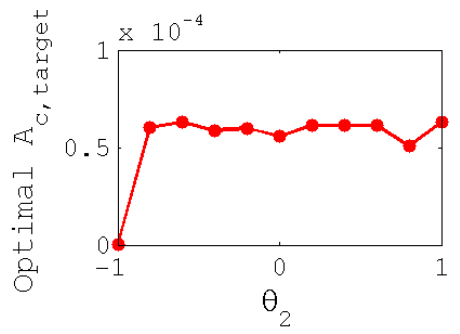

(b)

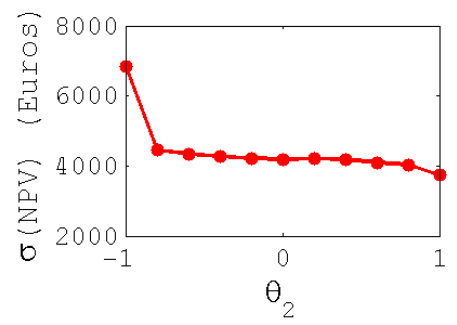

(e)

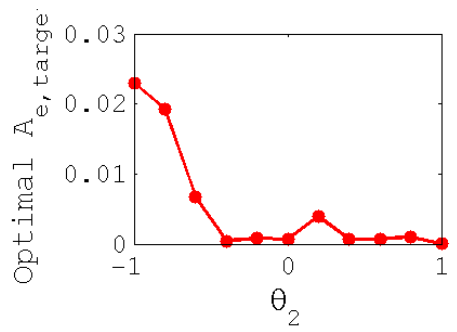

(c)

Figure 6.: Change in the parameters of the optimal design option, expected $N P V$, and standard deviation of $N P V$ with the weight on the standard deviation of the economic criterion distribution.

optimization scheme is shown in Table 4 and the associated $N P V$ distribution is shown in Figure 4. The solution is very similar to that obtained with the base-case scheme as it implicitly seeks solutions with a higher expected $N P V$ and a distribution with a lower dispersion to minimize the number of dwellings with a negative $N P V$.

\subsection{Impact of alternate optimization scheme}

Here we compare the impact of using an optimization scheme comprising of a multi-layered sampling scheme for generating the input variations and design options. The optimal design options considering an objective function constructed from an equally weighted sum of the expected value and standard deviation of the $N P V$ distribution $\left(S_{\mathrm{ml}}\right)$ and one constructed from $P(N P V>0)$ are shown in Table 4 and Figure 4. The solutions in both cases are almost indistinguishable from the hybrid genetic algorithm scheme, even though the preferred effective leakage area of the attic floor is a little lower in the former. This shows the potential insensitivity of the optimal design option to this parameter.

As the multi-variate space of the design options has been globally sampled, the Pareto-optimal front of the expected value and standard deviation of the NPV distribution can be reasonably well resolved (Figure 7). The curve bounding all the solutions shows the 'non-dominating' design options, ones for which an improvement cannot be obtained in one objective without adversely impacting the other. Discontinuous changes can be observed in this curve; this is most likely a result of the way maintenance costs are treated as a 'one-off' cost. The curve shows that for negative expected $N P V$ the mean increases while the standard deviation decreases, but this trend reverses for a positive expected $N P V$, i.e. they become conflicting for the risk-averse decision maker. 
Postprint: Das P, Van Gelder L, Janssen H, Roels S. 2017. Designing uncertain optimization schemes for the economic assessment of stock energy-efficiency measures, Journal of Building Performance Simulation, 10:3-16.

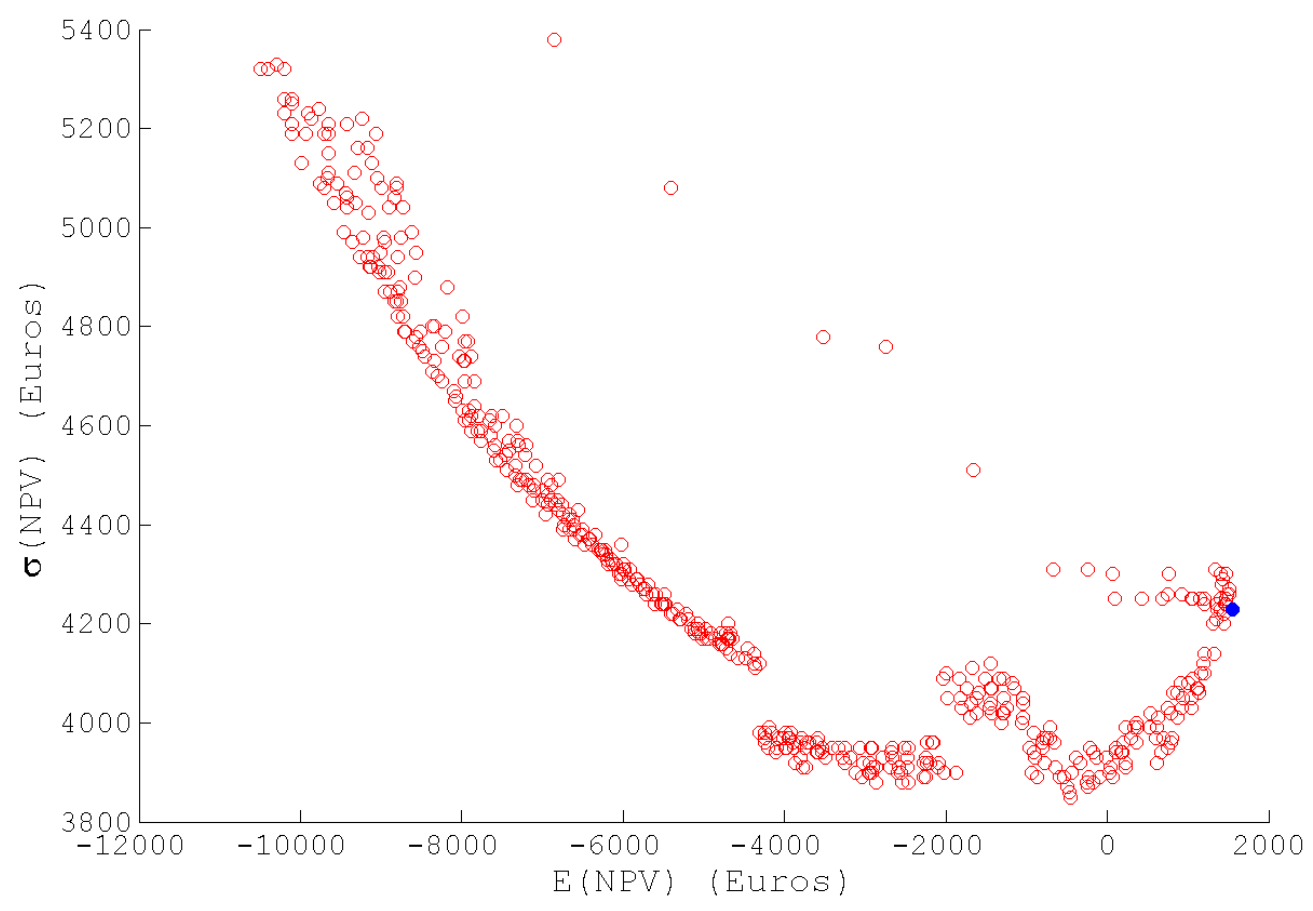

Figure 7.: Mean and standard deviations of $N P V$ for all the explored scenarios. The blue dot shows the optimal solution for an equal weight between the mean and the standard deviation, and for minimizing $P(N P V<0)$.

The sensitivities of the moments of the optimal $N P V$ distribution to the parameters of the design option can be easily assessed through the examination of scatter plots (Figure 8). The sensitivities are very clear and therefore can be assessed visually, without the requirement of a quantitative sensitivity analysis. The moments do not depend strongly on the target attic floor leakage area and to a very weak extent on the roof eave leakage area, consolidating the findings in Figure 6b) and c). However there is a strong sensitivity to the target $U$-value of the attic floor. There is a maximum in the expected value of $N P V$ at a target U-value of about $0.15-0.20$ that coincides with a minimum in $P(N P V<0)$, while the minimum in the standard deviation occurs at a target U-value of about 0.5 . Therefore $E(N P V)$ and $P(N P V<0)$ are not conflicting objectives and could be used interchangeably. The standard deviation prefers a different optimal U-value for a risk-averse decision maker, and therefore the trade offs between this objective and one of the others need to be examined in detail.

\section{Discussion}

In this section we discuss the optimal design options and how best to design an uncertain optimization scheme. We also allude to other variations in the scheme not explored in the paper.

\subsection{The optimal design option}

The optimal design options associated with a wide range of variations in economic criteria, formulation of the objective function, and method for generating design options all prefer a scenario in which both the U-value of the attic floor and effective leakage area in the attic roof eaves are lower than the current stock means, without much of a change in the effective leakage area of the attic floor. This shows that the heat transfer through conduction between the indoor living area 
Postprint: Das P, Van Gelder L, Janssen H, Roels S. 2017. Designing uncertain optimization schemes for the economic assessment of stock energy-efficiency measures, Journal of Building Performance Simulation, 10:3-16. doi: 10.1080/19401493.2015.1099054
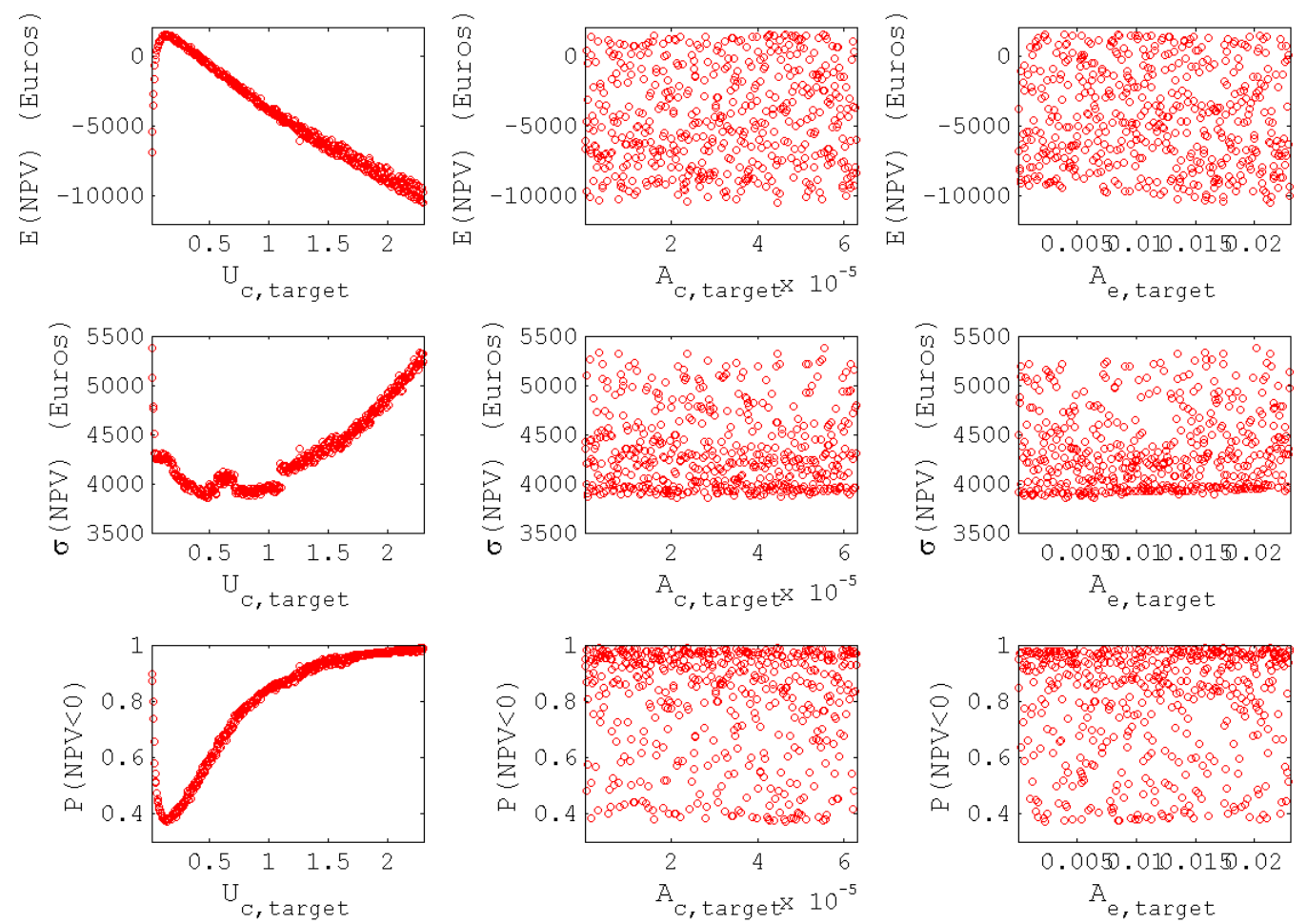

Figure 8.: Scatter plots of the mean, standard deviation, and $P(N P V>0)$ moments of the $N P V$ distributions against the parameters defining the design options.

and attic space, and heat and moisture transfer through ventilation between the attic space and outdoor environment should be minimized. This minimizes heat loss from the indoor living area to the attic floor through conduction, and heat loss from the attic to the outdoor environment through air exchange. Moisture ingress from the outdoor environment is also reduced. Ingress of moisture from the indoor environment to the attic is either less of an issue or, the ventilation rate from the indoor living area into the attic does not change appreciably, perhaps because the pressures are very similar in both spaces. The moments of the $N P V$ distribution are found to be most sensitive to the U-value of the attic floor and to some extent to the leakage area of the roof eaves, thus consolidating these findings.

\subsection{Why are there differences between economic criteria?}

Only $P B$ results in a noticeable impact on the optimal design option and equivalent $N P V$ distribution. The full benefit of a lower U-value on energy savings may not be fully realised over the shorter time periods probed by the payback period, particularly in the case of a low-cost design option. Another discrepancy is a lack of inclusion of the maintenance costs associated with mould damage. The criterion could be modified to include it, but in the current formulation of the maintenance cost, the costs to 'pay back' would jump at the point a maintenance cost is incurred, which may result in an undesired jump in the cumulative payback distribution. $P B$ also does not account for changes in the cost of energy and discount rates, thus potentially omitting the reality that in some dwellings there may be losses. ROI is a normalization of $N P V$ by the sum of the maintenance costs and investment costs. Therefore it does not affect the proportion of dwellings achieving a negative result for the same optimal design option, but it could affect the value of the dispersion compared to the mean. Therefore if both moments are used in the objective function, it could drive 
Postprint: Das P, Van Gelder L, Janssen H, Roels S. 2017. Designing uncertain optimization schemes for the economic assessment of stock energy-efficiency measures, Journal of Building Performance Simulation, 10:3-16. doi: 10.1080/19401493.2015.1099054

the optimization differently, but this is not observed in the case study presented here. IRR is the 'discount rate' that meets the maintenance and investment costs. Rather than normalizing $N P V$ this time, it normalizes the energy savings with respect to the maintenance costs and investment costs, and therefore is also a percentage. It also has the advantage of being annulized, i.e. it can be compared to IRR calculated for other projects calculated over a different time span. ROI cannot be annulized because energy costs change between years. The annulization results in a dispersion that is a smaller proportion of the mean, but this does not affect the derived optimal design option in this case study.

It is clear that $P B$ in this form is not in general suitable as a means of economic assessment. Choosing between $N P V, R O I$, and $I R R$ comes down to the nature of the retrofitting project. For example, if the costs are split between the homeowner and government, e.g. the investment costs can be borrowed from the government and then paid back through energy savings, as is done in the UK Green Deal ${ }^{3}$. The homeowner may then just be concerned with the absolute profit of the project, in which case $N P V$ would be most suitable. Alternatively, a housing association may be considering which collection of dwellings would be a better investment to retrofit, in which case $R O I$ and $I R R$ may be more suitable. If the government were trying to decide between investing in a nationwide refurbishment of homes or upgrading the rail system, the timescales of the projects may be very different and therefore they may only be interested in using $I R R$.

\subsection{How should the objective function be formulated?}

It is not obvious how to choose the ideal weighting between the mean and standard deviation of the performance criterion distribution a priori. However looking at a range of optimal solutions associated with several weights may help the decision maker make a more informed choice. For example, in the case study presented here, by examining different weights it could be seen that an optimal solution can be found with a similarly high $N P V$, but a noticeably lower dispersion by using a slightly higher weight on the standard deviation than used in the base-case scheme. In the case of economic criteria like $N P V, R O I$, and $I R R$, which can be negative, it may be sufficient to simply minimize the probability they are negative, and therefore avoid having to choose a weight between the mean and standard deviation. We only explored the use of $P(N P V<0)$ here, but one could also easily maximize $P(N P V>y)$, where $y$ is a target $N P V$ or minimize the probability of mould ever occurring.

\subsection{The importance of the optimization scheme}

The optimization scheme does not impact the optimal design option but there are several advantages and disadvantages with both the methods explored in this work. The hybrid genetic algorithm has the advantage of not having to choose the number of design options a priori, and therefore can be more computationally efficient at finding the optimal design option. It also samples more densely closer to the optimum, enabling us to better judge a range of design options with a similar degree of optimality in the context of a single-objective optimization problem. In the case where the exact form of the objective function is known (e.g. either the weight between the mean and standard deviation is known or $P(N P V<0)$ is used), this may be the most suitable method. However as the design parameter space is not fully explored as in the multi-layered sampling scheme, the same opportunities for further analysis are not available. For example, the Pareto-optimal front of the mean and standard deviation of the $N P V$ distribution is easily resolved if the design parameter space is globally sampled. This enables different solutions to be easily explored, without having to rerun the optimization, essentially allowing a multi-objective optimization to be performed. The random nature of the sampling of design options also enables a sensitivity analysis to be run to

\footnotetext{
${ }^{3}$ https://www.gov.uk/green-deal-energy-saving-measures/overview
} 
Postprint: Das P, Van Gelder L, Janssen H, Roels S. 2017. Designing uncertain optimization schemes for the economic assessment of stock energy-efficiency measures, Journal of Building Performance Simulation, 10:3-16. doi: 10.1080/19401493.2015.1099054

examine the sensitivity of the moments of the economic criterion distributions to parameters of the design options. This can also be done to some extent with the genetic algorithm iterations but the design space is not globally sampled.

Neither of the optimization methods explored here calculate the uncertainty on the optimal design parameters. A Bayesian method such as Markov Chain Monte Carlo, which samples the design option parameters according to the probability they are optimal, would be required to obtain a handle on the uncertainty. However, the methods explored here are much less computationally expensive, and are able to tell the decision maker, which design options would result in acceptable economic criterion distributions.

\section{Conclusions}

A wide number of combinations of economic criteria, objective function formulations, and methods of generating design options are explored in the context of determining the optimal retrofitting of cold attics in the Swedish climate, where a significant saving in the computational time required was made by the use of neural networks in place of the models. The case-study specification has been developed as part of the framework of the Annex 55 project of the IEA EBC programme. In the majority of combinations, the optimal design options prefer a lowering of the U-value of the attic floor and leakage area in the attic eaves, but no change in the leakage area of the attic floor. Therefore heat loss from the indoor living area, and both heat and moisture exchange between the attic and the outdoor environment are minimised. These changes would result in a $N P V$ distribution with a mean around $€ 1500$, a standard deviation around $€ 4200$, and a probability of 0.37 of dwellings only achieving a negative $N P V$. The moments of the $N P V$ distribution are found to be very sensitive to the U-value of the attic floor but less so to the leakage areas.

The payback criterion in the form explored here may be an unreliable indicator of the optimal design option, in particular in the case of a low-cost design option where it misses impacts in the time after the payback period, such as maintenance costs as a result of mould damage, or ongoing energy savings. It also does not account for changes in the cost of energy and how the energy savings may be discounted with time, but the criterion could be modified to do so. Though this is not currently standard in retrofitting projects, making these modifications would greatly improve the relevance of this criterion. The remaining forms of the economic criteria all account for maintenance costs, changes in energy costs, and a discount rate, and are directly comparable to each other as they are evaluated over the same time period. Choosing between them depends on whether the decision maker is more interested in absolute profit in which case they should use $N P V$. If they are more interested in the total fractional return for example in the case of retrofitting projects of different scales but the same life span, they should use ROI. In the case where the decision maker may be comparing projects of different scales and lifespans, the annulized fractional return or $I R R$ is most appropriate. The three criteria however result in the same optimal design option in the case study presented here.

Rather than choosing a single weight between the mean and standard deviation in the objective function formulation, it is preferrable to explore a range of weights and inspect the solutions corresponding to each one. Minimizing the probability that $N P V$ is lower than zero, gives very similar results to using approximately equal weights between the mean and standard deviation and may be a way of avoiding having to choose weights.

Using a hybrid genetic algorithm and a multi-layered sampling scheme to explore the parameter space of the design options give almost identical solutions. The hybrid genetic algorithm reaches the optimal design option efficiently and samples quite densely around the optimal solution to enable a range of similar solutions to be collected. However, the multi-layered sampling scheme allows for a higher degree of flexibility regarding subsequent analysis through the construction of alternative objective functions and sensitivity analyses. Although uncertainties on the optimal design parameters are not obtained, estimates of design options with similar impacts can be inferred, 
Postprint: Das P, Van Gelder L, Janssen H, Roels S. 2017. Designing uncertain optimization schemes for the economic assessment of stock energy-efficiency measures, Journal of Building Performance Simulation, 10:3-16. doi: 10.1080/19401493.2015.1099054

and with much less computational time compared to methods such as Markov Chain Monte Carlo.

\section{Acknowledgements}

PD would like to acknowledge funding from Engineering and Physical Sciences Research Council (EPSRC) grant EP/I02929X/1.

\section{References}

Ahrnens, C., Borglund, E., 2007. Fukt på kallvindar-en kartläggning av småhus i västra götalands län.

Asadi, E., da Silva, M. G., Antunes, C. H., Dias, L., 2012. A multi-objective optimization model for building retrofit strategies using TRNSYS simulations, GenOpt and MATLAB. Building and Environment 56 (0), $370-378$.

Booth, A., Choudhary, R., Spiegelhalter, D., Jan. 2013. A hierarchical Bayesian framework for calibrating micro-level models with macro-level data. Journal of Building Performance Simulation, 1-26.

Das, P., Chalabi, Z., Jones, B., Milner, J., Shrubsole, C., Davies, M., Hamilton, I., Ridley, I., Wilkinson, P., 2013. Multi-objective methods for determining optimal ventilation rates in dwellings. Building and Environment 66, 72-81.

Das, P., Nix, E., Chalabi, Z., Davies, M., Shrubsole, C., Taylor, J., 2014a. Exploring the health/energy pareto-optimal front for adapting a case-study dwelling in the delhi environment. In: Building Simulation Optimization.

Das, P., Shrubsole, C., Jones, B., Hamilton, I., Chalabi, Z., Davies, M., Mavrogianni, A., Taylor, J., 2014b. Using probabilistic sampling-based sensitivity analyses for indoor air quality modelling. Building and Environment 78, 171-182.

Deb, K., 2001. Multi-objective optimization using evolutionary algorithms. Vol. 2012. John Wiley \& Sons Chichester.

Fesanghary, M., Asadi, S., Geem, Z. W., Mar. 2012. Design of low-emission and energy-efficient residential buildings using a multi-objective optimization algorithm. Building and Environment 49 (1), 245-250.

Goodacre, C., Sharples, S., Smith, P., 2002. Integrating energy efficiency with the social agenda in sustainability. Energy and Buildings 34 (1), 53-61.

Hagentoft, C.-E., 2011. Probabilistic analysis of hygrothermal conditions and mould growth potential in cold attics. impact of weather, building system and construction design characteristics. In: Proceedings of International Conference on Durability of Building Materials and Components.

Hagentoft, C.-E., Kalagasidis, A. S., Nilsson, S., Thorin, M., Ventotech, A., 2008. Mould growth control in cold attics through adaptive ventilation. In: Proceedings of 8th Nordic Symposium of Building Physics.

Hopfe, C. J., Augenbroe, G. L., Hensen, J. L., 2013. Multi-criteria decision making under uncertainty in building performance assessment. Building and Environment 69 (0), 81 - 90.

Hukka, A., Viitanen, H., 1999. A mathematical model of mould growth on wooden material. Wood Science and Technology 33 (6), 475-485.

Janssen, H., 2013. Monte-carlo based uncertainty analysis: Sampling efficiency and sampling convergence. Reliability Engineering \& System Safety 109, 123-132.

Kuckshinrichs, W., Kronenberg, T., Hansen, P., Aug. 2010. The social return on investment in the energy efficiency of buildings in Germany. Energy Policy 38 (8), 4317-4329.

Malatji, E. M., Zhang, J., Xia, X., Feb. 2013. A Multiple Objective Optimisation Model for Building Energy Efficiency Investment Decision. Energy and Buildings 61 (1), 81-87.

Nguyen, A.-T., Reiter, S., Rigo, P., 2014. A review on simulation-based optimization methods applied to building performance analysis. Applied Energy 113 (0), 1043 - 1058.

Rysanek, A., Choudhary, R., 2013. Optimum building energy retrofits under technical and economic uncertainty. Energy and Buildings 57 (0), $324-337$.

Tuhus-Dubrow, D., Krarti, M., 2010. Genetic-algorithm based approach to optimize building envelope design for residential buildings. Building and Environment 45 (7), 1574-1581.

Turner, W. J., Logue, J. M., Wray, C. P., Feb. 2013. A combined energy and IAQ assessment of the potential value of commissioning residential mechanical ventilation systems. Building and Environment 60 (0), 194201. 
Postprint: Das P, Van Gelder L, Janssen H, Roels S. 2017. Designing uncertain optimization schemes for the economic assessment of stock energy-efficiency measures, Journal of Building Performance Simulation, 10:3-16.

Van Gelder, L., Das, P., Janssen, H., Roels, S., 2014a. Comparative study of metamodelling techniques in building energy simulation: Guidelines for practitioners. Simulation Modelling Practice and Theory 49, 245-257.

Van Gelder, L., Janssen, H., Roels, S., 2014b. Probabilistic design and analysis of building performances: methodology and application example. Energy and Buildings 79, 202-211. 